\title{
DESCRIPTION STRATEGIES FOR NAIVE AND EXPERT USERS
}

\author{
Cécile L. Paris \\ Department of Computer Science \\ Columbia University \\ New York, NY 10027
}

\begin{abstract}
It is widely recognized that a question-answering system should be able to tailor its answers to the user. One of the dimensions along which this talloring can occur is with respect to the level of knowledge of a user about a domain. In particular, responses should be different depending on whether they are addressed to naive or expert users. To understand what those differences should be, we analyzed texts from adult and junior encyclopedias. We found that two different strategies were used in describing complex physical objects to juniors and adults. We show how these strategies have been implemented on a test database.
\end{abstract}

\section{INTRODUCTION}

Whether the purpose of a natural language program Is to ease man-machine interactions (Kapian 82; Hayes and Reddy 79] or to model human communication [Lehnert 78], it must take into consideration certain characteristics of the person engaged in the interaction In an interaction between people, the goals, beliefs, intentions, knowledge and past expenence of the participants will play a role in how they communicate with each other [Cohen and Perrault 79], [Perrault and Allen 80|. Similarly, those characteristics should play a role in the way a computer system interacts with a user. In particular, a question-answering program that provides access to a large amount of data to many different users will be most useful if it can tailor its answers to each user.

We are interested here in how the level of knowledge (or expertise) of the user affects an answer. As an example of this kind of tailoring in a naturally occurning conversation, an explanation of how a car engine works aimed at a child will be different than one aimed at an aduit, and an explanation adequate for a music student is probably not quite sufficient for a student in mechanical engineering. In this paper, we study the strategies used in natural language to describe physical objects to two different types of users: naive and expert. By naive and expert, we refer to how famuliar a user is about the domain of the database as opposed to how experienced the user is with the question/answering system. When the database is complex, it becomes important to vary the level and the kind of details included in the answer in order to provide an answer that can be best understood by the user.
We plan to use this distinction in the questionanswering program for RESEARCHER, a system being developed at Columbia University. RESEARCHER reads, remembers, and generalizes from patents abstracts written in English [Lebowitz 83]. The abstracts describe complex physical objects in which spatial and functional relations are important. Thus, we are interested in characterizing spatial strategies that can be used for experts and novices about certan physical objects. We give details in the paper of the current implementation of description strategies on a test database of object descriptions.

\section{OUR DOMAIN}

Our goal is to characterize some of the strategies employed to describe complex physical objects and see whether these strategies are different for naive and experts users. To investigate this problem, we have looked at texts from encyclopedias (both adult and junior) and high school physics textbooks. The texts we have studied are about physical objects performing a lunction (such as telephones and telescopes), and generaily do not exceed several paragraphs in length. These texts make the distinction between naive and expert readers and have been widely used for a number of years for those audiences. They provide examples of different descriptive strategies that actually occur in natural language. Thus, a question-answering system should be able to reproduce them ${ }^{2}$

Studying texts from encyclopedias gives us the advantage of being able to compare descriptions of identical objects ammed at two distinct audiences. On the average, a younger audience has had less opportunity to gather experience and knowledge about any particular domain. Thus a younger audience as a whole is more naive about a domain than an adult audience. Hence, these texts give us a good starting point for studying the differences between the

\footnotetext{
${ }^{1} \mathrm{We}$ studied about fifteen examples from each encyclopedia and textbook.

${ }^{2}$ Our goal however is not to study how elfective these texts are for different buman readers. If further psychological research shows that other distinctions are appropnate, they could be incorporated. The distinction based on encyclopedias and textbooks is really the only available at this point.
} 
1) The hand-sets introduced in 1947 consist of a receirer and a transmitter in a single bousing available in black or colored plastic.

2) The transmitter diaphragm is clamped rigidly at its edges 3) to improve the high frequency response. 1) The diaphragm is coupied to a doubly resonant system 5) a cavity and an air chamber- 6) which broadens the response. 7) The carbon chamber contains carbon granules, 8) the contact resistance of which is varied by the diaphragm's vibration.

9) The receiver includes a ring-shaped magnet system arouad a coil and a ring shaped armature of anadium Permendur. 10) Current in the coil makes the armature vibrate in the air gap. 11) An attached phenolic-impregated fabrie diaphragm, shaped like a dome, 12) vibrates and sets the air in the canal of the ear in motion.

\author{
1. Constitueney \\ Depth-attributive for the tranamitter \\ (Description of the transmitter) \\ 2. Depth-Attributive \\ 3. Cause-etreet \\ 4. Depth-Attributive \\ 5. Depth-ideatification \\ 6. Cause-eftect \\ 7. Depth-Attributive \\ 8. Cause-effect
}

Depth-attributive for the receiver

(Description of the receiver)

\title{
9. Depth-Attributive
}

10. Cause-efreet

11. Attributive

12. Cause-efrect

Figure 1t Constituency Schema Example

descriptions given to naive users and those given to experts in the domain. To minimize the elfects of stylistic differences on our results, we chose texts from several different encyclopedias in each audience category.

\section{THE TEXTUAL ANALYSIS}

We began by analyzing the diffarent texts using methods developed by other researchers ( [Hobbs 78a], [Hobbs 80), (Mann 84], (McKeown 82]). We decomposed paragraphs in terms of therr primitive rhetorical structure ia $3 n$ attempt to find a consisteret structure in each group of texts. The analysis showed the adult encyclopedia descriptions to be mainly in terms of the sub-parts of the object being described. These texts can be characterized by one of the textual structures posited in [Mckeown 82], the constituency schema. This structure is presented in the next section. On the other hand, no schema or other organizing structure consistently accounted for the descriptions in the junior encyclopedia texts In lookıng lor other types of organizing strategies, we discovered that the main strategy in describing cbjects to a naive user is to trace through the process that allows the object to perform its lunction

\section{Strategy for the Adults}

The descriptions from the adult encyclopedias tend to follow the pattern established by the constituency schema, one of the textual structures defined in [Mckeown 82]. in her work on natural language generation, Mckeown studied the problems of what to say and how to organize text coherently. She examined texts and transcripts, classifying each sentence as one of a set of rhetorical predicates 3 and found that some combinations of predicates were more likely to occur than others. Moreover, for each discourse situation, some combination would be the most appropriate one. Those standard combinations were encoded as schemas which are associated with a particular discourse situation. One of these schemas is the constituency schema which is used to describe an object (or concept) in terms of its subparts and thejr properties. The constituency schema is shown below (For a given entity, Constituency is the description of its sub-parts or sub-types, and the attributive predicate gives properties associated with it.)

\footnotetext{
3Rhetorical predicates characterize the structural purpose of a sentence and have been discussed by a variety of linguists [Grimes 75] [Hobbs 78b] Some examples are conatituency (description of sub-parts or sub-types), attributive (providing detal about an entity or event) and analogy (the making of an analogy).

${ }^{4}$ We have altered McKeown's constituency schema slightly by making the first predicate optional instead of mandalory: in the texts studied, the main parts of the object were not necessarily immediately listed. We are using McKeown's notation:" \{\}$^{\prime \prime}$ indicate optionality, "I" Indicates alternatives, "+" Indicates that the item may appear 1-n times, and "wn indicates that the item may appear 0-n times. Finally, "n," is used to represent classification of ambiguous propositions.
} 
1. 1) When one speaks Into the transmltter of a modern telephone, these sound waves strike agalnst an aluminium disk or diaphragm and eause it to vlbrace back and forth in Just the same way the molecules of alr are vlbratlng.

II. 2) The center of this diaphragm is connected with the carbon button originally invented by Thomas A. Edison. 3) This is a little brass box filled with granules of earbon composed of especially selected and treated coal. 4) The front and back of the button are insulated.

III. 5) The talking curreat is passed through this box so that the electricity must nind its way from granule to granule inside the box. 0) When the dlaphragm moves inward under the pressure from the sound waves the carbon gralns are pushed together and the electrleity nnds an easler path. 7) Thus a strong current nows through the llne. 8) When a thin portion of the sound wave comes along, the diaphragm springs back, allowing the carbon particles to be more loosely packed, and eonsequently less eurrent ean nnd its way through. D) So a varying of undulating current is sent over the line whose vibrations exactly correspond to the vibraclons eaused by the speaker's volce. 10) This eurrent then nows through the llne to the coila of an electromegnet in the receiver.

\section{iV. 11) Very near to the poles of thie magnet is a thin iron dise.}

v. 12) When the current beeomes stronger it pulls the dise toward (t. 13) As a weaker current nows through the magnet, It is not strong enough to attract the disk and it springs back. 14) Thus the diaphragm in the recelver is made to vibrate in and out....

Figure 22 Text from a junior encyclopedia

\section{Constituencr Schema}

\section{\{Constituency\} \\ Cause-effect" / Attributive \\ \{ Depth-identification / Depth-attributive \{ Particular Illustration / Evidence \{Comparison, Analogy\} $\}^{+}$ \\ \{ Amplification / Explanation / Attributive / Analogy \}}

Consider for example the description of a telephone from an, adult encyclopedia [Collier 62] shown in Figure $1^{5}$ In the first sentence, the telephone is described in terms of its constituency (or sub-parts): the transmitter, the receiver and the housing. From sentence 2 to 8 , attributive information (or properties) as well as functional information (causeeffect) about the transmitter are given ${ }^{6}$ Finally, the receiver in turn is described from sentence 9 to 12 , using both attributive and cause-efreet information

${ }^{5}$ For clarity, the original one paragraph text has been divided into three paragraphs.

${ }^{6}$ The reader who is famliar with this type of analysis will note that several properties of the transmitter are in turn identified and described using altributive information which is a form of schema recursion.
Entries in the junior encyclopedia and high school text books

In texts aimed toward younger audiences, an object is mainly described in terms of the functions of its parts. The description traces through the process information instead of an enumeration of its sub-parts, as is usually the case in the adult descriptions. The parts are mentioned only when they need to be, that is, when the description of the mechanical process calls for them. As an example of this phenomenon, consider the description of $a$ telephone shown in Figure 2, taken this time from the encyclopedia junior [Britannica-Junior 63|

We see that the theme of this text is the mechanical process description shown in bold face. That process description gets interrupted when descriptive information can be included concerning a sub-part that was just mentioned as part of the process description. Such information is shown in indented italics in the example.

Furthermore, we see that, in the junior encyclopedia, not only is the description made mainly through a process trace, but there are no large gaps in

The original entry contained the two paragraphs. The second one has been divided for clarity 


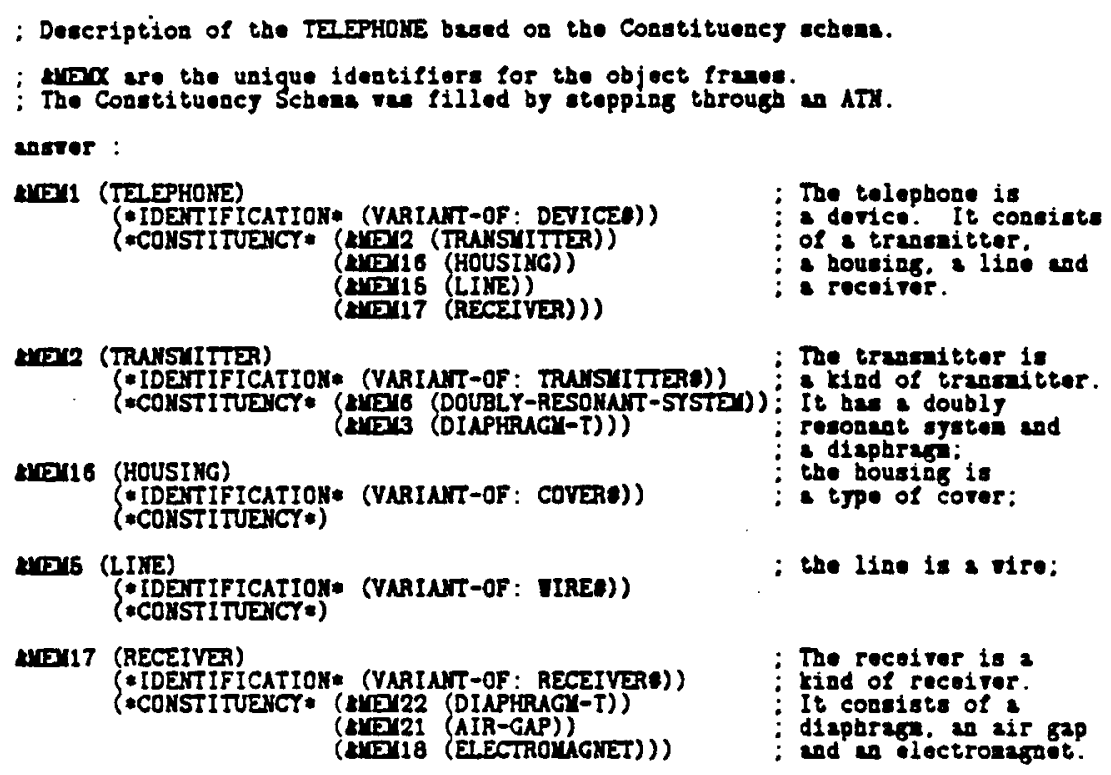

Figure 3t Printout of the Constituency Schema Example

the chain of inferences. Almost everything is spelled out. Consider the third paragraph of the text given above where every step is explanned:

"The talking current is passed through this box SO THAT the electricity must find its way FROM GRANULE TO GRANULE ingide the box."

From there, the wnter goes on to explain how the electricity passes through the carbon box, once again stepping through the process, spelling out the consequences of each step:

"When the diaphragm moves inward under the pressure from the sound waves the carbon grains are pushed together and the electricity finds an easier path. THUS a strong current nows through the line."

Contrast this detailed process description with the description given for an adult 8 .

"The carbon chamber contains carbon granules, the contact resistance of which is varied by the diaphragm's vibration".

Other differences occurred between the junior and adult entries as well. In general, more visual information was included in the text for the junior, so as to render the description more vivid. For example, the carbon button in the telephone description is described as "a !ittle brass box filled with carbon of especially selected and treated coal" in the junior

\footnotetext{
${ }^{8}$ This excerpt is taken from an adult encyclopedia.
}

encyclopedia, in contrast to "the carbon chamber contans granules" in the adult encyclopedia, similarly, the junior entry for light bulbs describes a filament as a "fine tungsten filament wound in very small cotls", whereas the adult encyclopedia mentions only "a colled tungsten filament."

Another major difference was that the junior encyclopedia texts had a higher degree of redundancy while the adult encyclopedia ones were quite concise. We refer to the junior telephone example again to lllustrate this point: sentences $j$ and 6 explained how the electricity is made to flow easily through the box Sentence 7 is a recapitulation of that phenomenon. Finally, sentence 8 explans the reverse effect

Finally, we observed that expository style and vocabulary differed considerably in the two types of texts studied. Future research will attempt to characterize these phenomena.

\section{COMPUTATIONAL USE OF THE STRATEGIES}

The strategies are currently implemented on a test database composed of object descriptions from the encyclopedias. The representation of an object thus contains all the information included for that particular object in both encyclopedias. The two 


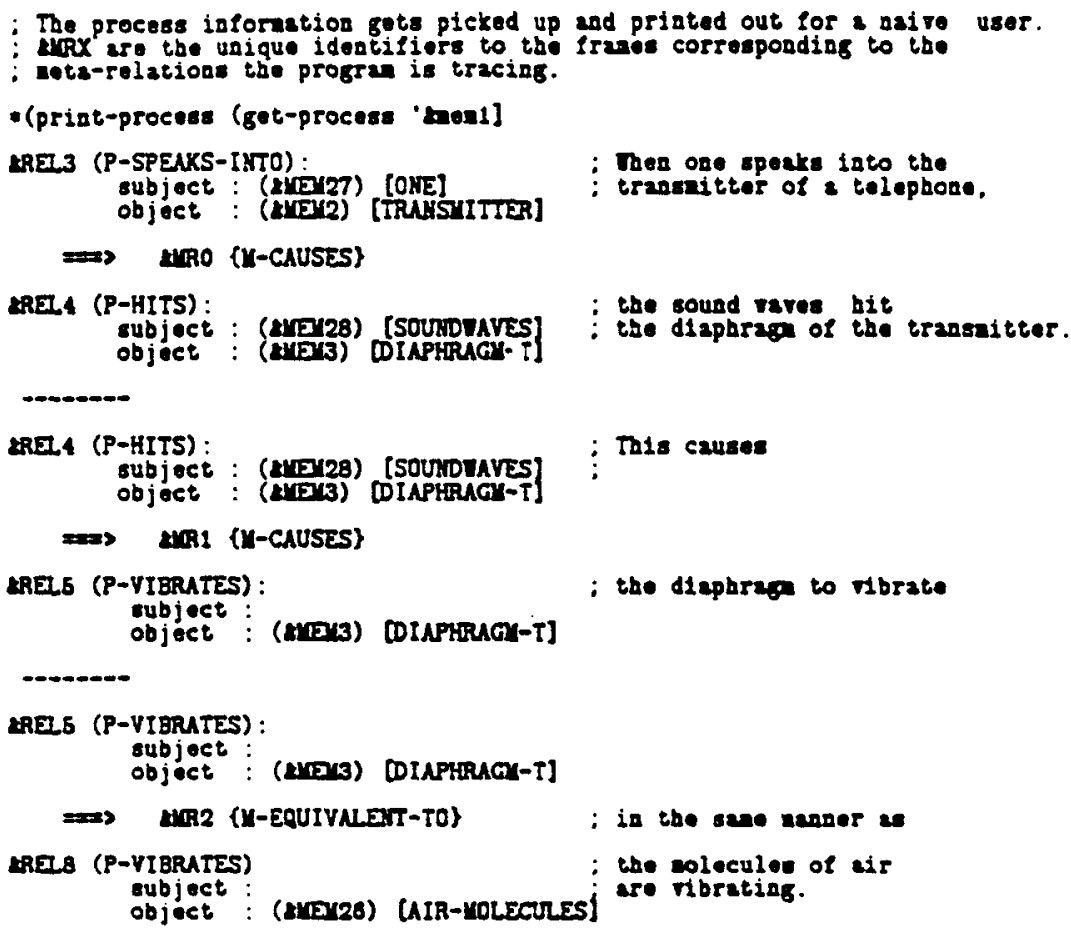

Figure 48 Printout of the Process Trace

strategies presented dictate what information to include from the knowledge base, based on the constituency schema or the process trace as shown in Figures 3 , 4 and $5^{9}$.

\section{Knowledge-based representation}

We use a frame-based knowledge representation [Wasserman and Lebowitz 83; Wasserman 85] in which the basic irame represents an object. These structures are the entities in a generalization hierarchy In addition to the generalization, or instance-of links, there exist two additional kinds of links joining entities: part-of links, which indicate an entity is a part of a larger structure, and relations, which convey information about spatial or functional relationships Finally, there are cousal links between relations called meia-relations

\footnotetext{
${ }^{9}$ Further work is needed to fully implement the schema predicates and add more descripuve information.
}

Implemention of the adult encyclopedia strategy

For an adult, the program fills the constituency schema, as shown in Figure 310 . The predicates contained in the schema define the type of information to be taken from the database. The figure shows the final output. The entities are represented by their unique identifier \&MEMLX, and the predicates are the starred items (e.g. "DENTIFICATION"). The hypothetical english output is included in the comments.

The identification predicate represents the more general concept of which the present object is in instance. Eecause the test database includes only the information contained in the texts read, the hierarchy may not be complete for all objects. As an example. a transmilter was never defined in terms of a more general device, and thus has no super-ordinate The constituency predicate gives the components of an entity, if there are any

\footnotetext{
${ }^{10}$ See [Mckeown 82] for details of a similar system
} 


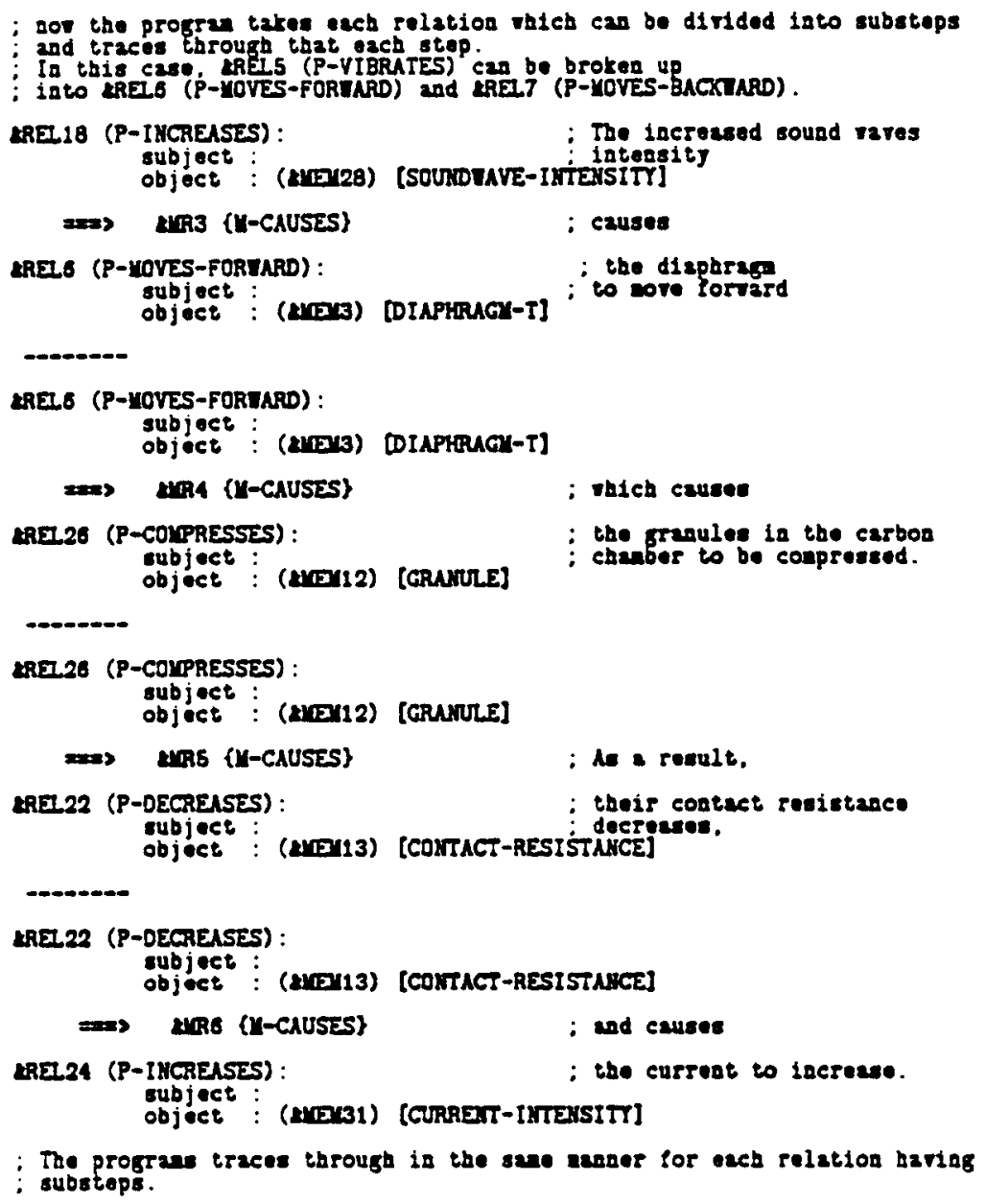

Flgure 6s Printout of the Process Trace (cont'd)

Junior encyclopedia strategy

For the junior, the strategy dictates to follow the cause-effects links in the knowledge base in order to trace the process. In our representation, these causual links are named meta-relations (In the figure they are represented by the identifiers \&:MRX \&RELX correspond to the relations, 1 e the spatial or functional links tetween entities). The program traces through the meta-rolations, picking the process information as shown in Fizure 4. When a relation can be broken into substeps, the program then traces through those sub-steps (see Figure 5)

\section{Future Work}

There are several theoretical issues that need to be addressed. In our test database, the problem of deciding in what order relations occur does not arise. However, for an arbitrary database, knowing where to begin describing $a$ process may be more difficult Simliarly, the process may not be as sequential as the ones we examined so lar, and, as a result, we plan on further study of how to organize the information. Furthermore, in our test database, we don't need to consider how deep into the substeps the process description should go, but this issue exists for an arbitrary database. Finally, we have looked at the two ends of a spectrum (naive and expert), but, for users not at either of these ends, we must consider how to combine these strategies. 
We have started to address the problem of generating natural language for the descriptions. We have begun the augmentation of an English surface generator [McKeown 82] that, using a functional grammar [Kay 79], takes the output of the textyal component to translate it into English sentences 1 . However, how this program may interface with the strategies remains to be studied.

\section{CONCLUSION}

It is important to tailor a system's response to the level of expertise of the user. By studying texts aimed at two different levels of readers, we have found that two different strategies were used in describing physical objects, depending on whether the description was for an adult or for a junior. For an adult, an object is described with its sub-parts and their properties; for a junior, the description traces through the mechanical process which renders the object functional.

The two strategies presented account for the main differences found between the adult and jumior entries. This turns out not to consist of merely giving more details for the expert as is often thought I $W$ allis and Shortliffe 82]. In the adult entries, details given are mainly about the sub-parts and their properties and less about the mechanical process involved. When the process mechanism is mentioned at all, it is done very briefly. In the junior entries, process mechanism is more important than sub-parts and given in more detail Parts are introduced either after or at the same time as their function is defined, and, as a consequence, are always defined when presented. Furthermore, since the process mechanism follows every step of the causal chain, descriptions for the novice tend to include more detal about functional information than descriptions for the expert. We have shown how formalization of the strategies allows for the development of question-answering systems which can tailor their responses to the user, given his level of expertise about the domain 12

\section{ACKNOWLEDGMENTS}

We would like to thank Kathy Mckeown and Michael Lebowitz for helping in both the research and the writing of this paper. This research was supported in part by the Defense Advanced Research Projects Agency under contract N00039-84-C-0165

\footnotetext{
${ }^{11}$ Determining the level of expertise of the user is another research problem which we have been studying ( $[$ Paris 84|).
}

12 Determining the level of expertise of the user is another research problem which we have been studying ( ( $\mathrm{P}$ aris 84]).

\section{References}

[Britannica-Junior 63]

Britannica Junior Encyclopedia

Encyclopedia Britannica Incorparation 1963; Wiliam Benton Publisher

[Cohen and Perrault 79]

Cohen, P. R. and Perrault, C. R

Elements of a Plan-Based Theory of Speech Acts.

Cognitive Science 3:177 - 212, 1979

[Collier 62]

Collier's Encyclopedia.

The Crowell-Collier Publishing Company 1962; William Halsey editorial director.

[Grimes 75] Grimes, J. E

The Thread of Discourse.

Mouton, The Hague, 1975

[Hayes and Reddy 79]

Hayes, $P$. and Reddy, $R$.

Graceful Interaction in Man-Machine Communication.

In Proceedings of the IJCAI. International Joint Conferences on Artificial Intelligence, 1979.

[Hobbs 78a] Hobbs, J.

Why is a Discourse Coherent?

Technical Report 176, SRI International, 1978.

[Hobbs 78b] Hobbs, J

Coherence and Coreference.

Technical Note 168, SRI International, 1978

Menlo Park, California.

[Hobbs 80] Hobbs, J. and Evans, D

Conversation as Planned Behavior

Cognitive Science 4(4):349 - 377, 1980

[Kaplan 82] Kaplan, S. J.

Cooperative Responses from a Portable Natural Language Query System. Artificial Intelligence 2(19), 1982.

[Kay 79] Kay, Martin.

Functional Grammar

In Proceedings of the 5th meeting of the Berkeley Linguistics Society. Berkeley Linguistics Society, 1979 
[Lebowitz 83] Lebowilz, M.

RESEARCHER: An Overview

In Proceedings of the Third National Conference on Artificial

Intelligence. American Association of Artificial Intelligence,

Washington, DC, 1983

[Lehnert 78] Lehnert, W. G.

The Process of Question Answering.

Lawrence Erlbaum Associates, Hillsdale, N. J., 1978

[Mann 84] Mann, W.C.

Discourse Structure for Text Generation

Technical Report ISI/RR-84-127, Information Sciences Institute, February, 1984

4676 Admiralty Way/ Marina del Rey/California 90292-669.5.

[Mckeown 82] McKeown, K.

Generating Natural Language Text in Response to Questions About Database Structure.

PhD thests, University of Pennsylvania May, 1982.

Also a Technical report, No MSCIS-82-05, University of Pennsylvania, 1982.

[Paris 84] Paris, C. L.

Determining the Level of Expertise.

In Proceedings of the First Annual Workshop on Theoretical lssues in Conceptual In formation Processing Atlanta, Georgia, 1984

[Perrault and Allen 80]

Perrauit R. C and Allen J. F

A Plan-Based Analysis of Indirect Speech Acts.

American Joumal of Computational Linguistics 6(3-4), 1980
[Wallis and Shortliffe 82]

Wallis, J.W. and Shortliffe, E.H.

Explanatory Power for Medical Expert Systems: Studies in the Representation of Causal Relationships for Clinical Consultation.

Technical Report STAN-CS-82-923, Stanford University, 1982.

Heuristics programming Project. Department of Medecine and Computer Science.

[Wasserman 85] Wasserman, $K$.

Unifying Representation and Generalization: Understanding Hierarchically Structured Objects.

PhD thesis, Columbia University Department of Computer Science, 1985.

[Wasserman and Lebowitz 83]

Wasserman, $K$. and Lebowitz, $M$. Representing Complex Physical Objects.

Cognition and Brain Theory 6(3) 333 - 352, 1983 